\title{
Ultrasonido endoscópico bronquial (EBUS): una herramienta extraordinaria en Broncoscopia Intervencionista
}

\author{
Bronchial endoscopic ultrasound, a new tool in \\ interventional bronchoscopy
}

Alejandra Cañas, MD(1); Diana Rey, MD(2); Carlos Celis Preciado, MD(3)

El desarrollo que ha experimentado la broncoscopia en los últimos cincuenta años puede considerarse admirable y acelerado. Sin duda, no existe otra área de la Neumología que haya logrado avances tan notorios, relevantes y profundos en tan poco tiempo.

En 1967, Shigeto Ikeda, en colaboración con la empresa Machida Endoscopic C. y Olympus Optical Co, diseñó un modelo de broncoscopio flexible que fue presentado en el IX Congreso Internacional de Neumología, celebrado en Copenhague, suscitando gran expectativa. Este fue el comienzo del desarrollo de la broncoscopia flexible y supuso un gran avance en la patología respiratoria al hacer posible la exploración de amplios territorios anatómicos del árbol bronquial, no accesibles con el broncoscopio rígido. Su introducción obligó a modificar la clasificación y nomenclatura de la distribución anatómica del árbol bronquial.

La realización de la broncoscopia y su tecnología han mejorado notablemente. Estamos en la generación número veinte de los broncoscopios flexibles, los canales de trabajo son más grandes, hay mejores accesorios, lo que posibilita la toma de biopsias de mayor tamaño, y hay algunos tan delgados como de 1,8 mm, con 3.000 fibras ópticas para la observación directa de objetos de 2 a $30 \mathrm{~mm}$ en un rango de 75 grados. El gran desarrollo tecnológico se base en el progreso de diferentes áreas: instrumentación, fibra óptica, fuentes de iluminación, electrónica y técnicas anestésicas, solo por mencionar algunas de las más sobresalientes.

El desarrollo del ultrasonido endoscópico (EBUS, del inglés EndoBronchial UltraSound) se remonta a una década, con Becker, basándose en los resultados obtenidos por los aparatos de ecoendoscopia digestiva en la estadificación de cánceres del tracto digestivo. En la actualidad, las aplicaciones diagnósticas del EBUS han aumentado de forma considerable pasando a ocupar un puesto relevante no sólo dentro de la especialidad, sino también en el campo de la cirugía torácica $(1,2)$.

\section{Ultrasonido endobronquial}

Como se mencionó, el desarrollo inicial de la aplicación endoluminal se hizo en Gastroenterología, pero la aplicación a través del esófago limita
${ }^{(1)}$ Internista, Neumóloga. Unidad de Neumología, Hospital Universitario San Ignacio. Pontificia Universidad Javeriana. Bogotá, Colombia.

${ }^{(2)}$ Fellow de Neumología. Pontificia Universidad Javeriana. Hospital Universitario San Ignacio. Bogotá, Colombia.

${ }^{(3)}$ Internista, Neumóloga. Unidad de Neumología, Hospital Universitario San Ignacio. Pontificia Universidad Javeriana. Bogotá, Colombia.

Correspondencia: Alejandra Cañas. Correo electrónico: alejandra.canas@javeriana.edu.co Recibido: 12-12-2013. Aceptado: 31-12-2013. 
la evaluación del mediastino inferior y lateral y, a partir de allí, se genera el desarrollo de tecnología para evaluación específica a través de la vía aérea central $(1,2)$.

A diferencia de la broncoscopia convencional, en la que la imagen se limita a la superficie de la mucosa y evalúa solo los cambios de la pared bronquial mediante signos indirectos, el EBUS ha permitido visualizar las capas de las paredes de las vías aéreas, determinar la relación entre los vasos y nódulos/masas extraluminales, guiar la biopsia de los ganglios linfáticos mediastinales e hiliares y estudiar las lesiones parenquimatosas periféricas $(1,2)$.

La obtención de imágenes por ultrasonido difiere completamente de los rayos X. Es la diferencia de la resistencia a las ondas del ultrasonido (impedancia) de los diversos tejidos la que genera la imagen, y dependerá en parte del contenido de agua. La aplicación externa del ultrasonido mediastinal es insuficiente para obtener imágenes; la limitación es la ventana acústica, por el contenido de aire del tejido pulmonar.

El transductor produce ondas de sonido, las cuales a su vez se reflejan; dado que el agua es un excelente conductor se utiliza un balón para la transmisión $\mathrm{y}$, finalmente, se produce una imagen de ultrasonido en dos dimensiones, a la cual se la puede hacer doppler, tomar medidas y realizar ajustes de contraste (figura 1).

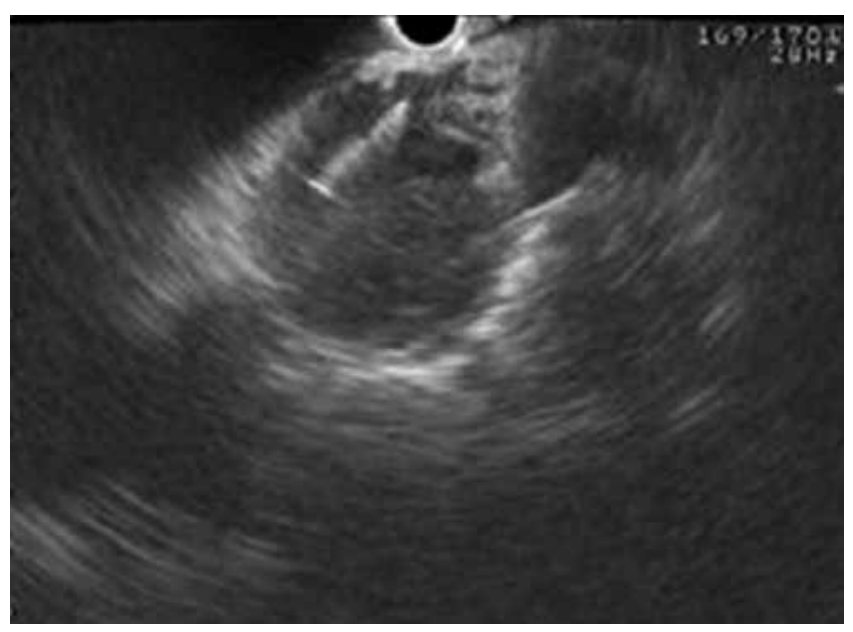

Figura 1. Imagen de EBUS.
En la última década se han desarrollado sondas ecográficas miniaturizadas que pueden ser insertadas a través de un conducto para el instrumental de 2,8 $\mathrm{mm}$. La punta de la sonda contiene un cristal piezoeléctrico que gira dentro de un balón lleno de agua, que cuando se infla, permite un acoplamiento de 360 grados con la vía respiratoria. El cristal giratorio funciona como generador de la señal y receptor simultáneamente. La frecuencia estándar del EBUS es $20 \mathrm{MHz}$, que permite una resolución de $<1 \mathrm{~mm}$ $\mathrm{y}$ una profundidad de la penetración de aproximadamente 4 a $5 \mathrm{~cm}$. Esta frecuencia permite una excelente evaluación de las capas de las paredes de las vías respiratorias y las estructuras parabronquiales. Las frecuencias más bajas (a 3,5 MHz) mejoran la profundidad de la penetración; sin embargo, se reduce la resolución espacial $(1,2)$.

Otro desarrollo relevante del EBUS es el conducto para el instrumental, pues permite imágenes durante punción aspiración transbronquial en tiempo real. Con el EVUS, lo que se ve en la pantalla de la imagen ecográfica, es la muestra que está tomando con la punción de aspiración transbronquial (TNBA, del inglés Transbronchial Needle Aspiration), y esto permite obtener muestras con alta sensibilidad y especificidad, como muestran los estudios (figuras 2 a 4) $(1,2)$.

Una de las ventajas del EBUS es la posibilidad de realizar el examen en forma ambulatoria, con mínima sedación, en general en corto tiempo, así como la evaluación de las muestras in situ y a costo menor. Sin embargo, debe tenerse presente que no está disponible universalmente y que en ocasiones la cantidad de tejido que se puede obtener es mínima y no es posible establecer un diagnóstico $(1,2)$.

\section{Manejo de las muestras}

El manejo de las muestras es un aspecto igual de crítico e importante, pues luego de realizar la aspiración, la aguja debe desocuparse en un recipiente con solución salina o alcohol al 95\%, evitar artefactos y transportarlo rápidamente al servicio de Patología para su interpretación $(1,2)$.

La evaluación rápida en el sitio de endoscopia de las muestras por el citopatólogo, incrementa las 


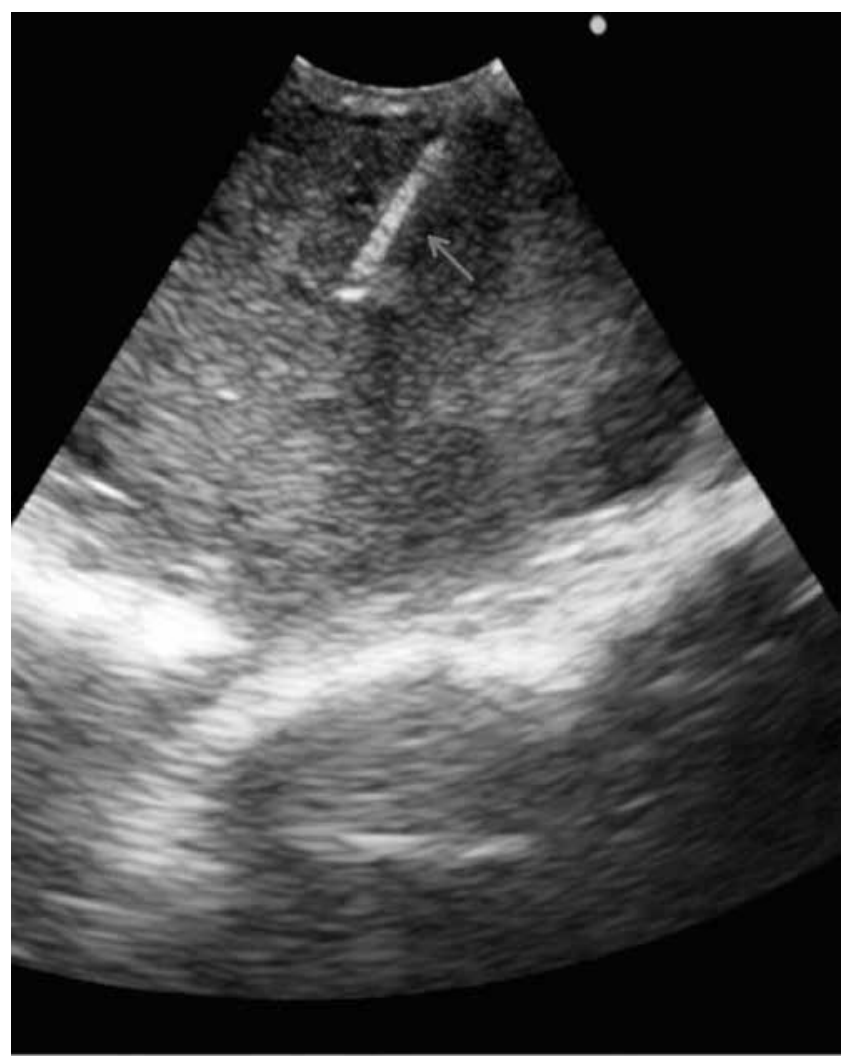

Figura 2. Visualización de estación ganglionar 10R a través de EBUS lineal y biopsia con aguja fina (flecha).

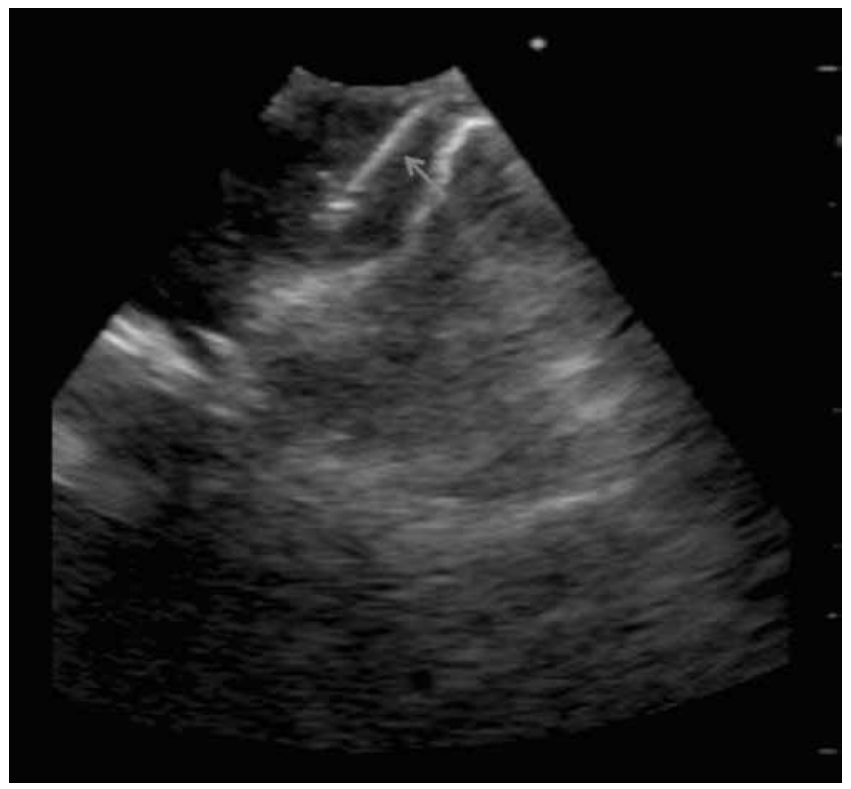

Figura 3. Visualización de estación ganglionar $10 \mathrm{R}$ a través de EBUS lineal y biopsia con aguja fina (flecha).

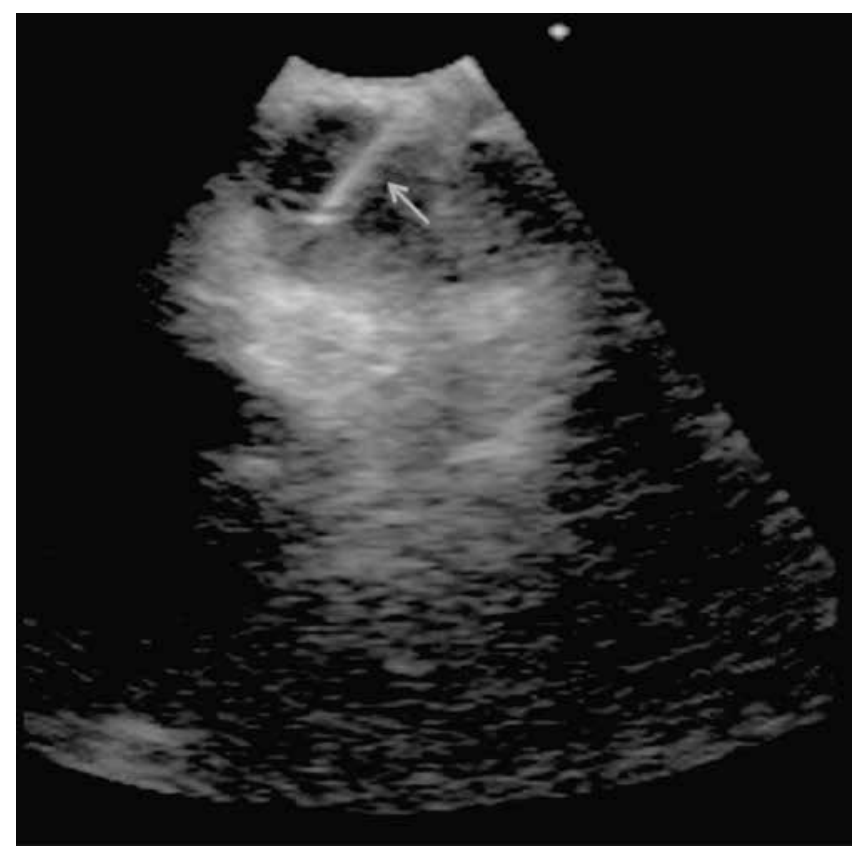

Figura 4. Visualización de estación ganglionar $4 \mathrm{~L}$ y toma de biopsia con aguja fina (flecha).

posibilidades de diagnóstico adecuado, por lo que el trabajo conjunto es fundamental a fin de obtener excelentes resultados con el estudio $(1,2)$.

Los criterios citológicos para considerar apropiadas las muestras obtenidas por AATB son la ausencia de células bronquiales y el predominio de linfocitos en la muestra examinada. Después de evaluar todas las muestras estas se clasificarán como negativas, sospechosas o malignas. Las muestras "verdaderamente" negativas son las que tienen presencia de linfocitos en ausencia de células malignas $(1,2)$.

\section{Indicaciones del uso del EBUS}

El EVUS se usa en la estadificación del cáncer de pulmón de células no pequeñas, en la evaluación diagnóstica de lesiones endobronquiales, nódulos pulmonares periféricos, alteraciones del mediastino (por ejemplo, linfadenopatías), y como ayuda en la terapia endoscópica (colocación de stents y braquiterapia), para distinguir entre la invasión de la vía respiratoria y compresión por tumor y determinar si el paciente podría beneficiarse de la resección quirúrgica. 


\section{Estadificación del cáncer broncogénico y extratorácico}

Una de las aplicaciones más estudiadas de esta tecnologia, es la estadificación del cáncer broncogénico, del cual dependerá el tratamiento y el pronóstico. El EBUS ha revolucionado la estadificación del $\mathrm{N}$ (presencia o no de compromiso neoplásico de los ganglios linfáticos) (3).

Se ha demostrado que el EBUS ayuda en la toma de muestra de ganglios mediastinales e hiliares, con un porcentaje de éxito global del $86 \%$, sin importar el tamaño o la localización del ganglio. Los ganglios detectados por EBUS pueden llegar a ser de 2 ó 3 $\mathrm{mm}$; hay estudios que reportan mejores rendimientos en EBUS-NTBA, hasta del $90 \%$. Esto es especialmente relevante en estaciones, como las 2 y 4 , donde puede errar la tomografía. Para la evaluación de la resecabilidad quirúrgica, la exclusión de N2 y $\mathrm{N} 3$ es lo relevante (3).

Sharples y colaboradores llevaron a cabo un estudio prospectivo de asignación aleatoria en cuatro centros europeos, con el fin de evaluar la eficacia de EBUS (seguido por estadificación invasiva si negativo) en comparación con mediastinoscopia en 241 pacientes con cáncer pulmonar de célula no pequeña candidatos a resección quirúrgica curativa (4).

La sensibilidad para la detección de metástasis N2/N3 fue de 79 (IC95\% 66-88) para la mediastinoscopia en comparación con 94\% (IC 95\% 85-98) para la estrategia de EBUS $(\mathrm{p}=0,02)$, con valores predictivos negativos del 86\% (IC95\%: 76-92) y del 93\% (IC95\%: 84-97; p=0,26), respectivamente. Se reportaron 21/118 (18\%) toracotomías innecesarias en el brazo de cirugía mientras que con EBUS fueron $9 / 123(7 \%)(p=0,02)$; no hubo diferencias en cuanto a complicaciones. El análisis de costo-efectividad del estudio mostró que la estrategia con EBUS era más económica que la de mediastinoscopia, con una diferencia media en el año de vida ajustado por calidad de vida, de 0,015 a favor de la ecoendoscopia (IC95\%: -0,023 a 0,052) (4).

Recientemente, Dong y colaboradores reportaron sensibilidad y especificidad del 90\% y del 99\%, , con valores predictivos positivo y negativo del $99 \%$ y del $93 \%$, respectivamente, tras realizar un meta-análisis de nueve estudios con 1.066 pacientes que habían recibido EBUS-TBNA para la evaluación del mediastino (5).

Por su parte, Yang y colaboradores evaluaron el valor del EBUS-TBNA para el diagnóstico de metástasis ganglionares intratorácicas en pacientes con neoplasias extratorácicas; la búsqueda (a mayo de 2014 y realizada en las bases de datos Cochrane, EMBASE y Medline) arrojó seis estudios (calidad moderada) con 533 pacientes. Los datos, obtenidos por análisis SROC de cada uno de los trabajos, mostraron sensibilidad del 85\% (IC95\% 95-100\%), especificidad del 99\% (IC95\% 95-100\%), LR+ 28 (IC95\% 11-71), LR0,16 (IC95\% 0,12-0,21), DOR 179 (IC95\%66-487) y área bajo la curva SROC 0,92 (6).

\section{Estudio de patología benigna}

La utilización de la biopsia por EBUS lineal en tiempo real ha posibilitado su uso en el diagnóstico de enfermedades no neoplásicas, con un rendimiento mayor sobre la base de los resultados obtenidos con las técnicas convencionales.

Específicamente en sarcoidosis, Agarwal y colaboradores han reportado, tras analizar la información de quince estudios con 553 pacientes, exactitud diagnóstica del 79\% (IC95\% 71-86), con solo cinco complicaciones menores (7).

Por su parte, Gupta y su equipo llevaron a cabo un estudio aleatorio en 130 pacientes con diagnóstico clínico de sarcoidosis con el fin de comparar EBUSTBNA con TBNA convencional, y demostraron mayor rendimiento diagnóstico con el primero $(74,5 \%$ vs. $48,4 \%$; $p=0,004)$, siendo aún más alto con el empleo conjunto de biopsias transbronquiales (8).

\section{Estudio de lesiones pulmonares periféricas}

Es frecuente encontrar lesiones pulmonares periféricas en la evaluación de los pacientes; por tanto, poder establecer un diagnóstico preciso es fundamental para evitar cirugías innecesarias, pero también se sabe que el estudio con técnicas convencionales tiene marcadas limitaciones $(9,10)$.

El EBUS ha incrementado de manera significativa el rendimiento diagnóstico en las lesiones pulmonares 
periféricas, independiente de tamaño de la lesión (8, 9). Steinfort y colaboradores, en un meta-análisis publicado en 2012, que evaluó los datos de dieciséis estudios con 1.420 pacientes, reportaron sensibilidad del 73\% (IC95\% 70-76\%), especificidad del 100\% (IC95\% 99-100\%), con LR+ de 26 (IC95\% 12-57) y LR- de 0,28 (IC95\% 0,23-0,36) (11).

Las lesiones de menos de $30 \mathrm{~mm}$ de diámetro con frecuencia no son visibles bajo fluoroscopia. Un estudio prospectivo evaluó el rendimiento diagnóstico de estas lesiones con EBUS. El 80\% de las lesiones de menos de $22 \mathrm{~mm}$ de diámetro pudo ser localizado con EBUS, y en el 70\% se estableció un diagnóstico con biopsia transbronquial. Se concluye, por ende, que el EBUS permite la orientación de la imagen como una alternativa a la fluoroscopia $(9,10)$.

\section{Estudio de la vía aérea}

La pared de la vía aérea puede ser examinada mediante EBUS radial y se pueden diferenciar hasta siete capas; la mucosa, el endocondrio, el pericondrio y la adventicia son hiperecoicas (blancas), mientras que la submucosa, el cartílago y la capa de tejido conectivo son hipoecoicas (grises).

El EBUS, así mismo, puede ayudar a guiar los procedimientos de Neumología Intervencionista, identificar las diferentes capas para guiar la colocacion de un stent, proceder a realizar dilataciones e identificar la malacia. También puede evitar provocar una hemoptisis fatal visualizando los vasos antes de un procedimiento de desbridamiento o ablación térmica.

\section{Conclusión}

El EBUS es una técnica diagnóstica mínimamente invasiva, indicada para el estudio de nódulos y ganglios mediastinales, así como para la estratificación mediastinal del carcinoma broncogénico. En algunos pacientes debe complementarse el estudio con otras técnicas como la mediastinoscopia. Es importante recalcar que los resultados positivos requieren un trabajo en equipo con el laboratorio de citopatología.

\section{Bibliografía}

Anantham D, Koh MS, Ernst A. Endobronchial ultrasound. Respir Med. 2009;103:1406-14.

Nakajima T, Yasufuku K, Yoshino I. Current status and perspective of EBUS-TBNA. Gen Thorac Cardiovasc Surg. 2013;61:390-6.

Fielding DI, Kurimoto N. EBUS-TBNA/staging of lung cancer. Clin Chest Med. 2013;34:385-94.

Sharples LD, Jackson C, Wheaton E, Griffith G, Annema JT, Dooms C, et al. Clinical effectiveness and cost-effectiveness of endobronchial and endoscopic ultrasound relative to surgical staging in potentially resectable lung cancer: results from the ASTER randomised controlled trial. Health Technol Assess. 2012;16:1-75.

Dong X, Qiu X, Liu Q, Jia J. Endobronchial ultrasound-guided transbronchial needle aspiration in the mediastinal staging of non-small cell lung cancer: a meta-analysis. Ann Thorac Surg. 2013;96:1502-7.

Yang B, Li F, Shi W, Liu H, Sun S, Zhang G, Jiao S. Endobronchial ultrasound-guided transbronchial needle biopsy for the diagnosis of intrathoracic lymph node metastases from extrathoracic malignancies: A meta-analysis and systematic review. Respirology. 2014 Jun 17. [Epub ahead of print]

Agarwal R, Srinivasan A, Aggarwal AN, Gupta D. Efficacy and safety of convex probe EBUS-TBNA in sarcoidosis: a systematic review and metaanalysis. Respir Med. 2012;106:883-92.

Gupta D, Dadhwal DS, Agarwal R, Gupta N, Bal A, Aggarwal AN. Endobronchial Ultrasound Guided TBNA vs. Conventional TBNA in the diagnosis of sarcoidosis. Chest. 2014 Jan 3. [Epub ahead of print]

Schuhmann M, Eberhardt R, Herth FJ. Endobronchial ultrasound for peripheral lesions: a review. Endosc Ultrasound. 2013;2:3-6.

Kurimoto N, Miyazawa T, Okimasa S, et al. Endobronchial ultrasonography using a guide sheath increases the ability to diagnose peripheral pulmonary lesions endoscopically. Chest. 2004;126:959.

Steinfort DP, Khor YH, Manser RL, Irving LB. Radial probe endobronchial ultrasound for the diagnosis of peripheral lung cancer: systematic review and meta-analysis. Eur Respir J. 2011;37:902-10. 